“ (C) 2016 IEEE. Personal use of this material is permitted. Permission from IEEE must be obtained for all other uses, in any current or future media, including

reprinting/republishing this material for advertising or promotional purposes, creating new collective works, for resale or redistribution to servers or lists, or reuse of any copyrighted component of this work in other works." 


\title{
Scattering Analysis of Asymmetric Metamaterial Resonators by the Riemann-Hilbert Approach
}

\author{
Piotr M. Kamiński ${ }^{1}$, Richard W. Ziolkowski ${ }^{2}$, Samel Arslanagić ${ }^{3}$ \\ ${ }^{1,3}$ Department of Electrical Engineering, Electromagnetic Systems, Technical University of Denmark \\ Ørsteds Plads, Bldg. 348, DK-2800 Kgs. Lyngby, Denmark \\ e-mail:pmarka@elektro.dtu.dk \\ 2 Department of Electrical and Computer Engineering, University of Arizona \\ 1230 E. Speedway Blvd. Tucson, Arizona, 85721-0104
}

\begin{abstract}
This work presents an analytical treatment of an asymmetric metamaterial-based resonator excited by an electric line source, and explores its beam shaping capabilities. The resonator consists of two concentric cylindrical material layers covered with an infinitely thin conducting shell with an aperture. Exact analytical solution of the problem is derived; it is based on the $n$-series approach which is casted into the equivalent Riemann-Hilbert problem. The examined configuration leads to large enhancements of the radiated field and to steerable Huygens-like directivity patterns. Particularly, the directivities can be reshaped by changing the source location, as well as the extent of the aperture. The present results may provide alternative strategies for beam shaping and may contribute to development of novel nano-antenna designs.
\end{abstract}

\section{INTRODUCTION}

Notable efforts over the past decade have allowed significant progress in the area of metamaterials (MTMs) and their applications. Conventional double-positive (DPS) materials may be combined with those exhibiting negative material parameters to achieve, e.g., sub-wavelength resonators and scatterers [1-3], waveguides, invisibility cloaks and antennas [4]. In these works, it was analytically shown that MTM-based canonical structures, despite being electrically small, can be super-resonant and lead to, e.g., immense enhancements of the field radiated by a source in their vicinity. Specifically, a strong electric dipole mode can be excited in a symmetric circularly cylindrical MTM-based configuration [2, 3]. The strength of this, and other modes can be effectively controlled, thus providing a possibility of tailoring their directivity [3]. Attempts to enhance the directivity of related configurations at optical frequencies have likewise been made $[5,6]$, where the analysis was performed by pure numerical means. In these works, high directivities were achieved through appropriate perforations of the scatterers, resulting in asymmetric shapes required for the excitation of higher order modes to boost their directivities.

Inspired by the recent efforts on enhancing the directivity of small particles in the optical domain $[5,6]$, it is presently of interest to examine how potential asymmetries in the cylindrical MTM-based configurations from [2, 3] may affect their resonant properties and thus their directivities. The purpose of this work is to examine, by purely analytical means, an example of such an asymmetric resonator loaded with
MTMs. The examined configuration consists of two, infinitely long, concentric cylindrical material layers covered by an infinitely thin, and perfectly electrically conducting (PEC) infinite cylindrical shell with an aperture in it. The structure is excited by an arbitrarily located electric line source (ELS). The asymmetry in the configuration is achieved by introducing an aperture in the PEC shell, whose extent is arbitrary. An exact analytical solution to this problem is derived. The so-called $n$ series approach is employed and is reformulated in terms of the equivalent Riemann-Hilbert problem known in the complex variable theory. This enables straightforward and efficient determination of the resulting near- and far-field properties. These include quantities such as the radiated power, nearfields, and associated power flow densities, as well as the power spectrum and directivity. Theoretical designs exhibiting simultaneously Huygens-like directivity patterns, partial beamsteering, and notable enhancements of the radiated fields will be presented and discussed as functions of the ELS location and the aperture extent. In the framework of the analytical analysis of asymmetric scatterers, it is worth noting that the present work is an extension of the studies in [7-10], where plane wave scattering from and coupling to an infinite metallic circular cylinder with an infinite axial slot which encloses a concentric infinite impedance cylinder was considered.

Throughout the work, the time factor $\exp (j \omega t)$, with $\omega$ being the angular frequency and $t$ being the time is assumed and suppressed.

\section{CONFIGURATION}

The cross section of the studied configuration is shown in Fig. 1. A circular cylindrical core of radius $a$ (Region 2) is covered with a concentric cylindrical layer of outer radius $b$ (Region 1). An infinitely thin PEC shell of radius $b$ with an aperture of total angular extent $2 \theta$ concentrically covers both material layers. The structure is immersed in free-space; hence, Region 0 has the permittivity $\varepsilon_{0}$, permeability $\mu_{0}$, and wavenumber $k_{0}=\omega \sqrt{\varepsilon_{0} \mu_{0}}$. Region $i, i=1$ and 2 , has the permittivity $\varepsilon_{i}$, permeability $\mu_{i}$, the wavenumber $k_{i}=\omega \sqrt{\varepsilon_{i} \mu_{i}}$, and the intrinsic impedance $\eta_{i}=\sqrt{\mu_{i} / \varepsilon_{i}}$. The structure is excited by an infinitely long ELS; a constant current $I_{0}$ flows on it. The axis of the cylinders and the ELS are parallel. A cylindrical coordinate system $(\rho, \phi, z)$ and the associated Cartesian coordinate system $(x, y, z)$ are introduced such that 
the $z$-axis coincides with the axis of the cylinders. The coordinates of the ELS are $\left(\rho^{\prime}, \phi^{\prime}\right)$ while those of the observation point are $(\rho, \phi)$. The aperture lies in the angular region $-\theta<\phi<\theta$. Analytical analysis discussed in the next section is general, but the ELS location is considered only in Region 2 due to the space limitations.

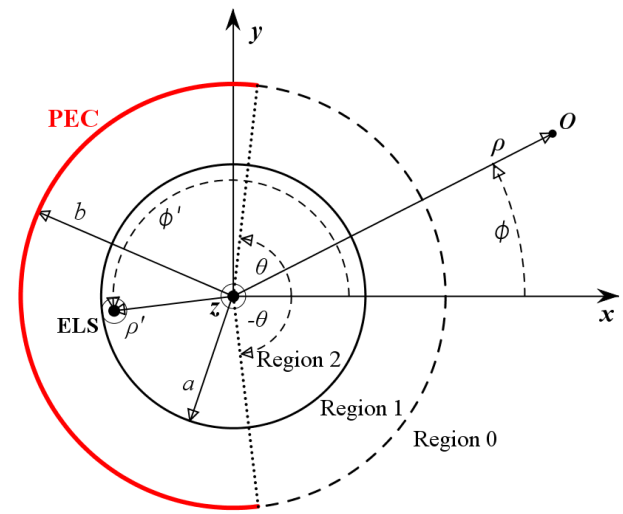

Fig. 1 A PEC shell with an aperture that is loaded with two concentric cylindrical material layers and that is excited by an ELS, which is depicted here as being located in the inner material layer.

\section{THEORY}

\section{A. Reduction to the Dual Series Problem}

The electric field radiated by the ELS is given by

$$
\bar{E}_{\mathrm{ELS}}^{\mathrm{inc}}=\hat{z} \frac{I_{0} k_{2} \eta_{2}}{-4} \sum_{m=-\infty}^{\infty} e^{j m\left(\phi-\phi^{\prime}\right)}\left\{\begin{array}{l}
J_{|m|}\left(k_{2} \rho\right) H_{|m|}^{(2)}\left(k_{2} \rho^{\prime}\right), \rho \leq \rho^{\prime} \\
H_{|m|}^{(2)}\left(k_{2} \rho\right) J_{|m|}\left(k_{2} \rho^{\prime}\right), \rho \geq \rho^{\prime}
\end{array}(1)\right.
$$

where $J_{|m|}(\cdot)$ is the Bessel function of order $m$, chosen to represent the field for $\rho \leq \rho^{\prime}$ due to its non-singular behavior at the origin, and where $H_{|m|}^{(2)}(\cdot)$ is the Hankel function of the second kind and order $m$, chosen for $\rho \geq \rho^{\prime}$ since it represents an outward propagating circular wave. Similarly, the electric fields associated with the structure are expanded in the same manner as:

$$
\bar{E}=\left\{\begin{array}{cc}
\hat{z} \frac{I_{0} k_{2} \eta_{2}}{-4} \sum_{m=-\infty}^{\infty} A_{m} J_{|m|}\left(k_{2} \rho\right) e^{j m \phi}+\bar{E}_{\mathrm{ELS}}^{\mathrm{inc}}, & \rho<a \\
\hat{z} \frac{I_{0} k_{1} \eta_{1}}{-4} \sum_{m=-\infty}^{\infty}\left(B_{m} J_{|m|}\left(k_{1} \rho\right)+C_{m} H_{|m|}^{(2)}\left(k_{1} \rho\right)\right) e^{j m \phi}, & a<\rho<b \\
\hat{z} \frac{I_{0} k_{0} \eta_{0}}{-4} \sum_{m=-\infty}^{\infty} D_{m} H_{|m|}^{(2)}\left(k_{0} \rho\right) e^{j m \phi}, & \rho>b
\end{array}\right.
$$

with $A_{m}, B_{m}, C_{m}$, and $D_{m}$ being the unknown expansion coefficients. The magnetic fields corresponding to the electric fields in (1) and (2) are easily obtained through the MaxwellFaraday law, and are not included in here. In order to solve the problem, the boundary conditions are applied. First, the continuity of the tangential electric and magnetic fields is imposed at the boundary $\rho=a$. The number of unknown coefficients is reduced further by requiring the continuity of the tangential component of the electric field across the interface $\rho=b$. At this point the total fields can be expressed solely in terms of a single modal coefficient, in our case $B_{m}$. In order to obtain the dual series representation, two boundary conditions are finally applied at $\rho=b$ : the condition for the tangential component of the electric field at the PEC shell, and the continuity of the tangential component of the magnetic field across the aperture. With this at hand, auxiliary coefficients are introduced and the dual series equations can be shown to take on the following form [7]

$$
\begin{aligned}
& \sum_{m=-\infty}^{\infty} g_{m} e^{j m \phi}=0,|\phi|>\theta \\
& \sum_{m=-\infty}^{\infty} g_{m} \tau_{m} e^{j m \phi}=f(\phi),|\phi|<\theta
\end{aligned}
$$

where

$$
\begin{aligned}
& g_{m}=B_{m}\left(J_{|m|}\left(k_{1} b\right)-\Omega_{|m|} H_{|m|}^{(2)}\left(k_{1} b\right)\right)+H_{|m|}^{(2)}\left(k_{1} b\right) \psi_{m}, \\
& \tau_{m}=\frac{J_{|m|}^{\prime}\left(k_{1} b\right) H_{|m|}^{(2)}\left(k_{0} b\right)-\frac{\eta_{1}}{\eta_{0}} H_{|m|}^{\prime(2)}\left(k_{0} b\right) J_{|m|}\left(k_{1} b\right)}{-j \pi H_{|m|}^{(2)}\left(k_{0} b\right)\left(J_{|m|}\left(k_{1} b\right)-\Omega_{|m|} H_{|m|}^{(2)}\left(k_{1} b\right)\right)}+ \\
& +\frac{\frac{\eta_{1}}{\eta_{0}} H_{|m|}^{\prime(2)}\left(k_{0} b\right) H_{|m|}^{(2)}\left(k_{1} b\right) \Omega_{|m|}-\Omega_{|m|} H_{|m|}^{\prime(2)}\left(k_{1} b\right) H_{|m|}^{(2)}\left(k_{0} b\right)}{-j \pi H_{|m|}^{(2)}\left(k_{0} b\right)\left(J_{|m|}\left(k_{1} b\right)-\Omega_{|m|} H_{|m|}^{(2)}\left(k_{1} b\right)\right)}, \\
& \Omega_{|m|}=\frac{\frac{k_{1} \eta_{1}}{k_{2} \eta_{2}} J_{|m|}\left(k_{1} a\right)-\frac{k_{1}}{k_{2}} \frac{J_{|m|}\left(k_{2} a\right)}{J_{|m|}^{\prime}\left(k_{2} a\right)} J_{|m|}^{\prime}\left(k_{1} a\right)}{\frac{k_{1} \eta_{1}}{k_{2} \eta_{2}} H_{|m|}^{(2)}\left(k_{1} a\right)-\frac{k_{1}}{k_{2}} \frac{J_{|m|}\left(k_{2} a\right)}{J_{|m|}^{\prime}\left(k_{2} a\right)} H_{|m|}^{\prime(2)}\left(k_{1} a\right)}, \\
& \psi_{m}=\frac{\frac{2 j e^{-j m \phi^{\prime}} J_{|m|}\left(k_{2} \rho^{\prime}\right)}{\pi k_{2} a J_{|m|}^{\prime}\left(k_{2} a\right)}}{\frac{k_{1} \eta_{1}}{k_{2} \eta_{2}} H_{|m|}^{(2)}\left(k_{1} a\right)-\frac{k_{1}}{k_{2}} \frac{J_{|m|}\left(k_{2} a\right)}{J_{|m|}^{\prime}\left(k_{2} a\right)} H_{|m|}^{\prime(2)}\left(k_{1} a\right)}, \\
& f(\phi)=\sum_{m=-\infty}^{\infty} f_{m} e^{j m \phi}, \\
& f_{m}=\tau_{m} \psi_{m} H_{|m|}^{(2)}\left(k_{1} b\right) \\
& +\psi_{m} \frac{\frac{\eta_{1}}{\eta_{0}} H_{|m|}^{\prime(2)}\left(k_{0} b\right) H_{|m|}^{(2)}\left(k_{1} b\right)-H_{|m|}^{(2)}\left(k_{1} b\right) H_{|m|}^{(2)}\left(k_{0} b\right)}{-j \pi H_{|m|}^{(2)}\left(k_{0} b\right)} .
\end{aligned}
$$

In the above equations, a prime denotes the derivative with respect to the entire argument. To the best of our knowledge, the results in (3) and (4), which have the form of a dual series problem, have not been obtained before. They are next reformulated into an equivalent Riemann-Hilbert (RH) problem, from which the solution is obtained.

\section{B. Equivalent Riemann-Hilbert Problem}

The $\mathrm{RH}$ problem is concerned with finding a sectionally analytic function $x$ satisfying a prescribed transition condition across an open or a closed contour. Its limits $x_{+}$and $x_{-}$from the inside and outside of a closed curve satisfy the transition condition $x_{+}=g x_{-}+h$ on an open segment of the curve. The problem and its solution are described thoroughly in [11], and its relationship to the aperture coupling problem, as the one 
depicted in Fig. 1, is discussed in [7]. To obtain the equivalent $\mathrm{RH}$ problem, the expression (3a) is differentiated with respect to $\phi$ and $x_{m}=g_{m} m$ is then substituted into (3a) and (3b). The following functions, analytic on the interior and exterior of the contour, are introduced next

$$
x_{+}(z)=\sum_{m>0} x_{m} z^{m}, \quad x_{-}(z)=-\sum_{m<0} x_{m} z^{m} .
$$

Substituting these in (3a) and (3b), the dual series problem can be rewritten in the form equivalent to the inhomogeneous $\mathrm{RH}$ problem as

$$
x_{+}\left(e^{j \phi}\right)=T\left(e^{j \phi}\right) x_{-}\left(e^{j \phi}\right)+F\left(e^{j \phi}\right)
$$

where

$$
\begin{gathered}
T\left(e^{j \phi}\right)=\left\{\begin{array}{cc}
1 & \phi \in I(L) \\
-1 & \phi \in I(\Gamma)
\end{array}\right. \\
F\left(e^{j \phi}\right)=\left\{\begin{array}{cc}
0 & \phi \in I(L) \\
\xi g_{0}+f(\phi)-\sum_{m \neq 0} x_{m} \frac{|m|}{m} \chi_{m} e^{j m \phi} & \phi \in I(\Gamma)
\end{array} .\right.
\end{gathered}
$$

The factor $T$ is the coefficient of the Riemann-Hilbert problem, whereas $F$ is its free term. $\boldsymbol{I}(\Gamma)$ and $\boldsymbol{I}(L)$ denote the angular decomposition of the interval $[0,2 \pi]$ corresponding to the two complementary sets, $\Gamma$ and $L$. Subsequently, as shown in [8] and [10], the application of the so-called Sochocki's formula leads to an infinite linear system of equations

$$
\begin{array}{cl}
x_{m}=\xi V_{m}^{0} g_{0}+\sum_{n=-\infty}^{\infty} f_{n} V_{m}^{n}-\sum_{n=-\infty}^{\infty} \operatorname{sign}(n) x_{n} \chi_{n} V_{m}^{n}+2 \sum_{j=0}^{n-2} c_{j} R_{m-j}, & m \neq 0 \\
0=\xi V_{0}^{0} g_{0}+\sum_{n=-\infty}^{\infty} f_{n} V_{0}^{n}-\sum_{n=-\infty}^{\infty} \operatorname{sign}(n) x_{n} \chi_{n} V_{0}^{n}+2 \sum_{j=0}^{n-2} c_{j} R_{-j}, \quad m=0 \\
-g_{0}=\xi W_{l}^{0} g_{0}+\sum_{n=-\infty}^{\infty} f_{n} W_{l}^{n}-\sum_{n=-\infty}^{\infty} \operatorname{sign}(n) x_{n} \chi_{n} W_{l}^{n}+2 \sum_{j=0}^{n-2} c_{j} S_{l}^{j}, l=1, \ldots, n-1
\end{array}
$$

with

$$
\begin{aligned}
V_{n}\left(e^{j \phi}\right) & =\frac{1}{\pi i} \int_{\Gamma} \frac{G(\tau) \tau^{n} d \tau}{\tau-e^{j \phi}}, \quad V_{m}^{n}\left(e^{j \phi}\right)=\frac{1}{2 \pi} \int_{\Gamma} \frac{V_{n}\left(e^{j \phi}\right) e^{-j m \phi} d \phi}{G\left(e^{j \phi}\right)} \\
R_{m} & =\frac{1}{2 \pi} \int_{\Gamma} \frac{e^{-j m \phi} d \phi}{G\left(e^{j \phi}\right)}, \quad S_{l}^{j}=\sum_{m \neq 0} \frac{R_{m-j}}{m} e^{j m \psi_{l}} \\
W_{l}^{n} & =\sum_{m \neq 0} \frac{V_{m}^{n}}{m} e^{j m \psi_{l}}, \quad G(z)=\prod_{k=1}^{n-1}\left(z-a_{k}\right)^{\frac{1}{2}}\left(z-b_{k}\right)^{\frac{1}{2}} .
\end{aligned}
$$

where $z$ is a complex variable, and $a_{k}$ and $b_{k}$ are points of discontinuity on the contour. The solution of the system (7) yields the coefficients, $x_{m}$ and $x_{n}$. The coefficients (8a)-(8c) were derived for the circular contour with a single aperture and can be found in [8]. When the single aperture case is considered, i.e, $n=2$, those coefficients are combinations of Legendre polynomials. Once $x_{m}$ is found, $g_{m}$ can be determined $\left(x_{m}=g_{m} m\right)$ and consequently $B_{m}$, see (4a). It is noted that the solution in [10] is designed to provide an unbounded solution at the points of discontinuity, which are the ends of the contour $\Gamma$. Therefore, the boundary condition for the field, which possesses a discontinuous $\phi$ component, has to be cast into an equation considered over $I(I)$, the angular region in (3b). Thus, for TM polarized waves, the aperture is considered over the angular region $I(I)$ and the PEC constitutes the complementary region. The system (7) was implemented in Matlab and the resulting solution was verified with COMSOL Multiphysics. Excellent agreement was obtained, as will be shown in the presentation.

\section{RESULTS AND DISCUSSION}

It was shown in [2] that electrically small MTM-based structures with positive and negative material parameters can be designed to possess resonances, in contrast to their DPSonly counterparts. Therefore, DNG material parameters were chosen for Region 2, i.e., $\varepsilon_{2}=-\varepsilon_{0}$ and $\mu_{2}=-\mu_{0}$. Region 1 was free-space. Consequently, we refer to this case as the DNGDPS configuration. Enhancements of the radiated field are expected in this case due to the impedance matching between the different regions. To identify a resonance, the power ratio (PR) (power radiated by the ELS with the structure normalized with the power radiated by the ELS alone) is obtained as the radius of the outer layer is varied. The PR values were generated for several aperture extents, $2 \theta$. In our investigations, the frequency of operation was arbitrarily set to $f_{0}=300 \mathrm{MHz}$. The inner radius was set to $a=6 \mathrm{~mm}$. The aperture was defined by $\theta=45^{\circ}$. The maximum PR: $51 \mathrm{~dB}$, was produced when the ELS is close to the core boundary at $\rho^{\prime}=$ $5.99 \mathrm{~mm}, \phi^{\prime}=180^{\circ}$, and the outer radius $b=49.6 \mathrm{~mm}$. The resulting electric field, power flow density, directivity, and the power spectrum for this maximum case are shown in Fig. 2.

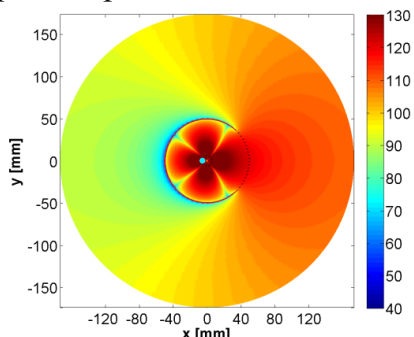

(a)

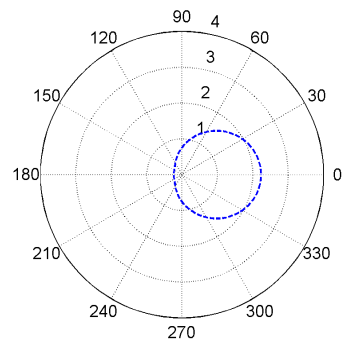

(c)

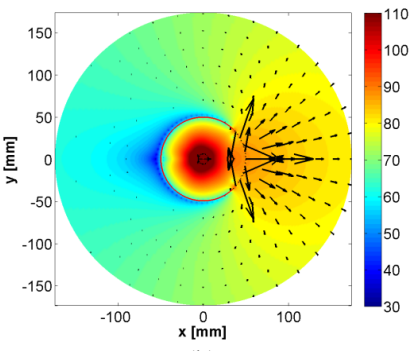

(b)

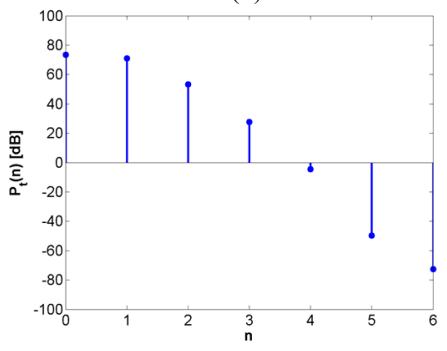

(d)
Fig. 2 Results for the DNG-DPS structure excited by an ELS. (a) Magnitude (dB) of the electric near-field, (b) power flow density, (c) directivity, and (d) power spectrum. The inner radius $a=6.0 \mathrm{~mm}$, the outer radius $b=49.6 \mathrm{~mm}$, and the aperture angle $\theta=45^{\circ}$.

The electric field inside the PEC shell has a distinct quadrupolar character, see Fig. 2(a). This is not the case outside of it since only one of the poles can radiate through the aperture. The power flow shown in Fig. 2(b) is strongly localized in the forward direction, but also has some power leaking to its sides. The latter is a result of the asymmetry in the structure which leads to higher order modes being generated and to a corresponding strong localization of the power flow in the near-field. On the other hand, the directivity 
pattern shown in Fig. 2(c) is produced by combinations of several modes; this is illustrated with the power spectrum values given in Fig. 2(d) for the monopole, dipole, quadrupole and sextupole modes.

The impact of different angular positions of the ELS on the the PR values and the directivity patterns were also studied. These results are shown in Fig. 3.

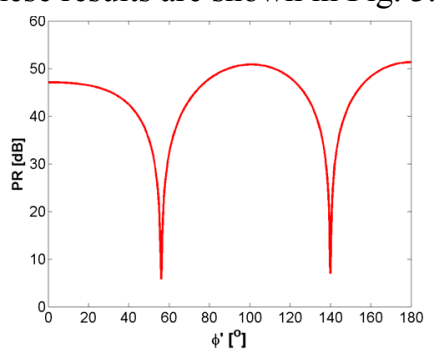

(a)

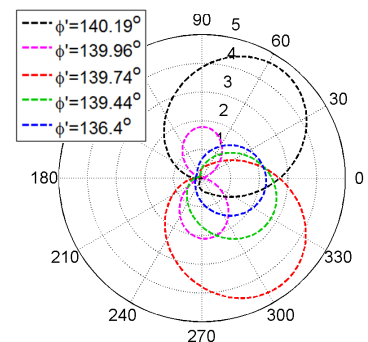

(b)
Fig. $3 \operatorname{PR}\left(\phi^{\prime}\right)$ for the structure in Fig. 2. The directivities for the ELS locations close to the second minimum in (a) are shown in (b).

The directivity pattern associated with each minimum in Fig. 3(a) is primarily dipolar. On the other hand, a Huygenslike directivity pattern is found just before $\left(\phi^{\prime}=139.74^{\circ}\right)$ and after $\left(\phi^{\prime}=140.19^{\circ}\right)$ where each PR minimum occurs, e.g., at $\phi^{\prime}$ $=139.74^{\circ}$ and $140.19^{\circ}$ for the minimum at $\phi^{\prime}=139.96^{\circ}$. For these two cases, the directivity is $\sim 4.7$ and the PR is $\sim 9 \mathrm{~dB}$. Thus, a small change in the angular location of the ELS can lead to a significant change in the directivity behavior of the beam. Moreover, Fig. 3(b) also indicates that for ELS locations further away from the minimum, e.g., $\phi^{\prime}=139.44^{\circ}$, the directivity drops significantly, below 3 , and approaches a monopolar pattern at $\phi^{\prime}=136.4^{\circ}$. When the asymmetric PEC shell is not present, it was observed in [2] that a change in the angular position of the ELS leads to a mere rotation of dipolar pattern. Thus, the enhanced Huygens-like patterns for these configurations are only obtained with the presence of the open PEC shell. The corresponding DPS-based structure does not lead to any resonance phenomena nor any high directivities, as will be illustrated in our presentation.

In order to design a configuration exhibiting some beam steering accompanied by a high enhancement of the radiated fields, structures with larger apertures were considered. Minima in the PR values were expected to be wider and shallower for these cases, thus allowing beam steering without significant loss in the PR. The radius of the outer layer was swept for various combinations of $\phi^{\prime}$ and $\theta$. As shown in Fig. 4, a desirable Huygens-like directivity pattern was obtained for $\theta=90^{\circ}$ with the $\mathrm{PR}=37 \mathrm{~dB}$. The maximum of the directivity pattern given in Fig. 4(b) is around 3.5 and no back-lobes are in evidence. It is noted that the back-lobe is smallest when the ELS is located near the aperture edge just inside the PEC shell, as illustrated in Fig. 4(a). The beam can also be partially steered by varying the ELS angular position near the PR minimum. For the Fig. 4 case, one finds the PR value to be decreased by only $8 \mathrm{~dB}$ compared to the PR maximum. Taking into account that the $P R$ values are generally much higher in this case, a difference of only $8 \mathrm{~dB}$ is considered to be relatively small. In the presentation, we will also show that the directivity can be reshaped by merely changing the angular extent of the aperture. Additional configurations including dispersion and loss, which deteriorate the performance of the structure, will also be presented.

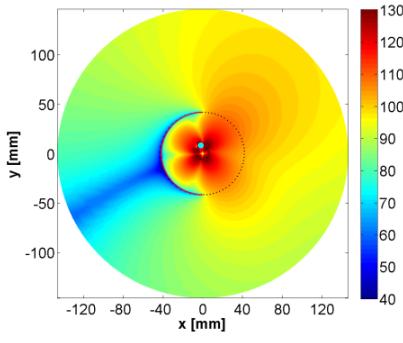

(a)

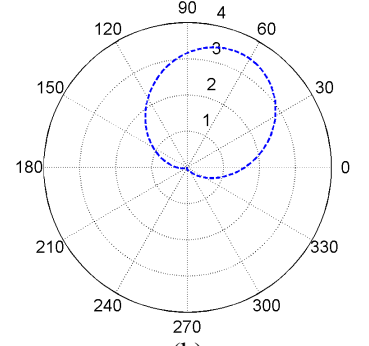

(b)
Fig. 4 Magnitude (dB) of the electric near field (a), and the directivity (b) for the DNG-DPS structure with $b=41.74 \mathrm{~mm}$ and $\theta=90^{\circ}$; the ELS is located at $\rho^{\prime}=5.99 \mathrm{~mm}, \phi^{\prime}=103.33^{\circ}$.

\section{CONCLUSION}

In this paper, we have presented an analytical treatment of asymmetric metamaterial-based resonators excited by an electric line source. The beam shaping capabilities of various cases were emphasized. The examined configurations lead to large enhancements of the radiated field as well as steerable Huygens-like directivity patterns. The directivities can be reshaped by changing the source location, as well as the extent of the aperture. These results may provide alternative strategies for beam shaping and may contribute to development of novel nano-antenna designs.

\section{REFERENCES}

[1] N. Engheta, R. W. Ziolkowski, "A positive future for double-negative metamaterials", IEEE Trans. Microw. Theory Techn., vol. 53, no. 4, pp. 1535-1555, 2005.

[2] S. Arslanagić, R. W. Ziolkowski, O. Breinbjerg, "Analytical and numerical investigation of the radiation and scattering from concentric metamaterial cylinders excited by an electric line source", Radio Science, vol. 42, RS6S15, 2007.

[3] S. Arslanagić and R.W. Ziolkowski, "Directive properties of active coated nano-particles," Adv. Electromag., vol. 1, no. 1, pp. 57-64, 2012.

[4] A. Alù, N. Engheta, "Enhanced directivity from subwavelength infrared/optical nano-antennas loaded with plasmonic materials or metamaterials", IEEE Trans. Antennas Propag., vol. 55, no. 11, pp. 3027-3039, 2007.

[5] S. D. Campbell, R. W. Ziolkowski, "Near-field directive beams from passive and active asymmetric optical nanoantennas", IEEE Journal of Selected Topics in Quantum Electronics, vol. 21, no. 4, August 2015.

[6] A. E. Krasnok, C. R. Simovski, P. A. Belov, Y. S. Kivshar, "Superdirective dielectric nanoantennas", Nanoscale, vol. 6, no. 13, pp. 7354-7361, 2014.

[7] R. W. Ziolkowski, W. A. Johnson, K. F. Casey, "Applications of Riemann-Hilbert problem techniques to electromagnetic coupling through apertures", Radio Science, vol. 19, no. 6, pp. 1425-1431, 1984.

[8] W. A. Johnson, R. W. Ziolkowski, "The scattering of an H-polarized plane wave from an axially slotted infinite cylinder: A dual series approach", Radio Science, vol. 19, no. 1, pp. 275-291, Jan. 1984.

[9] R. W. Ziolkowski, J. B. Grant, "Scattering from cavity-backed apertures: the generalized dual-series solution of the concentrically loaded Epolarized slit cylinder problem", IEEE Trans. Antennas Propag., vol. AP-35, no. 5, pp. 504-528, 1987.

[10] R. W. Ziolkowski, " $N$-series problems and the coupling of electromagnetic waves to apertures: a Riemann-Hilbert approach", SIAM J. Math. Anal., in press, 1984.

[11] F. D. Gakhov, Boundary Value Problems, Pergamon, London, 1966. 